\title{
Moxa Tar, an important source for developing new anti- inflammatory drug
}

\author{
Qian Zeng* \\ Medi7 Clinic Bentleigh, Australia
}

\begin{abstract}
Chronic inflammatory diseases are common and seriously affect people's life, like COPD, Asthma, inflammatory bowel diseases, especially atherosclerosis related diseases (heart attack, stroke and peripheral blood vessel diseases). Many chronic inflammatory diseases are resistant to current anti-inflammatory drugs, like COPD and atherosclerosis related diseases, therefore there is an urgent need to develop new, disease modifying anti-inflammatory drugs. Moxa (Artemisia Argyi) is a plant. Moxa tar (or moxa smoke) is the burning product of its leaves. From clinical experiments, the author finds that Moxa tar has potent and unique anti-inflammatory effect. Its anti-inflammatory property is different from that of any current anti-inflammatory drugs. It not only can suppress chronic inflammation but also can terminate self-perpetuating chronic inflammation which is the underlying mechanism of many incurable inflammatory diseases (like COPD). The author thinks that moxa tar is an important source for developing new anti-inflammatory drug and would like to introduce its anti-inflammatory effect to researchers through his clinical experiments in this article. The methods used in this study are crossover design, i.e., patients serve as their own control, comparing the clinical symptoms and signs before and after moxa tar treatment.
\end{abstract}

\section{Introduction}

Chronic inflammatory diseases are common and seriously affect people's life, like COPD, Asthma, atherosclerosis related diseases (heart attack, stroke and peripheral blood vessel diseases), inflammatory bowel diseases, chronic muscle and soft tissue injury and so on.

Many chronic inflammatory diseases are resistant to current antiinflammatory drugs, like COPD and atherosclerosis related diseases and therefore there is an urgent need to develop new anti-inflammatory drugs. The current strategy of developing new anti-inflammatory drugs is to target and block certain step or steps in the inflammatory passway via competitive inhibition. Some new anti-inflammatory drugs have been developed, like phosphodiesterase-4 inhibitors, NF-KB inhibitors, adhesion molecule inhibitors, tumour necrosis factor-alpha inhibitors, leukotriene B4 inhibitors, interleukin I receptor inhibitors and so on. Some of these new drugs can suppress chronic inflammation and alleviate clinical symptoms to some extent, but hardly have any curative effect for chronic inflammation.

From clinical practising, the author finds that moxa tar (or moxa smoke), a product of burning moxa leaves (Artemisia Argyi leaves), has potent and unique anti-inflammatory effect and it can treat many intractable chronic inflammatory diseases, like COPD, chronic muscle and soft tissue injury, chronic gastritis, angina pectoris, chronic laryngitis, thromboangiitis obliteran (Buerger's disease) and pulmonary fibrosis. Its anti-inflammatory property is different from that of corticosteroid and other known anti-inflammatory drugs. It not only can treat some corticosteroid resistant chronic inflammation, but also can cure self-perpetuating chronic inflammation. If the active antiinflammatory ingredients of moxa tar can be identified and isolated to make a new drug and used systemically, it will revolutionize the treatment of chronic inflammatory diseases. But so far, moxa tar is alien to western medical field and its therapeutic value is unknown to drug developers. The author would like to introduce the anti-inflammatory effect of moxa tar to molecular biologists who are interested in developing new drugs.

Artemisia Argyi, also called moxa or Chinese mugwort, is an herbaceous perennial plant. It is native to China, Korea, Mongolia, Japan and the Russian Far East. Its leaves have been used to treat various diseases by Chinese people for more than two thousand years.

A traditional Chinese therapy using moxa is called moxibustion. It is to grind dried moxa (Artemisia Argyi) up to fluff and further process it into a cigar-shaped stick or cone. Practitioners burn this moxa stick to heat certain points (acupuncture points) on the patient's skin or put the moxa cones on the acupuncture points and burn them to treat various diseases.

This therapy has two thousand and five hundred years history in China (it is also practiced in Japan and Korea), but it is little known to western society. It is highly regarded by traditional Chinese herbalists claiming it can treat hundreds of different diseases, including many intractable cases, but its therapeutic value has been unfairly neglected since western medicine came into China. One of the reasons is that its mechanism is not clear.

Chinese herbalists think moxibustion produces a special radiating heat which can penetrate the skin, give the body extra energy, warm the blood and open the body's blocked meridian lines ${ }^{1}$ to improve the blood flow to treat diseases. This explanation is based on the theory of Traditional Chinese Medicine. This theory is neither understood nor

Correspondence to: Qian Zeng, Medi7 Clinic Bentleigh, Australia, E-mail: qianzng@yahoo.com.au

Key words: corticosteroid, moxa tar or moxa smoke, moxibustion, atherosclerosis, COPD (chronic obstructive pulmonary disease), phosphodiesterase- 4 inhibitors

Received: April 10, 2017; Accepted: April 25, 2017; Published: April 28, 2017 
accepted by Western medical practitioners.

The author has carefully studied moxibustion for many years believing that the major therapeutic effect of moxibustion is due to the strong and special anti-inflammatory effect of the moxa tar produced by the burning moxa. The role of the heat in moxibustion is to improve the local blood circulation and dilate blood vessels and hair follicles, facilitating absorption of the moxa tar through the skin. The heat produced by burning moxa per se does not have anti-inflammatory effect or special therapeutic effect just as the heat produced by other methods. Without moxa tar moxibustion does not have any therapeutic effect.

The author has done experiments, using foil to cover the acupuncture points on the skin of patients and then heated them with burning moxa stick. The heat produced by burning moxa stick can penetrate the foil to heat the skin of the patients, but the moxa tar cannot reach the skin of the patients. After many treatments with moxibustion, these patients did not have any improvement in the conditions they were treated for. The other experiment is to collect moxa smoke in water. The moxa smoke becomes moxa tar when it reaches the water and then dissolves in the water. The author applied this moxa-tar-dissolved-water to eczematous lesions of patients, the eczema was cured. This experiment proves that moxa tar still has anti-inflammatory effect without the heat.

\section{Treatment of self-perpetuating chronic inflammatory diseases with moxa tar}

\section{Treatment of chronic laryngitis with moxa tar}

Chronic laryngitis is usually caused by upper respiratory tract virus infection, voice abuse, heavy smoking or alcohol consumption. The initiating causes can be eliminated, but the inflammation is persistent because prolonged inflammation causes tissue damage (damage to the wall of capillary and post capillary venule could change the permeability of these blood vessels, resulting in constant leucocytes recruitment), therefore the inflammation becomes self-perpetuating. Chronic laryngitis is not a life threatening disease, but it is an incurable disease, especially the severe cases. The chronic inflammation in chronic laryngitis is resistant to any known anti-inflammatory medications, including glucocorticoid steroid.

The author suffered from very severe chronic laryngitis secondary to flu

In year 2000. He had severe pain, dryness and heaviness (swelling) in the larynx. Initially he was treated with oral Prednisolone 50mg daily for 4 weeks, but his condition did not respond to steroid treatment at all. Then the author tried moxibustion therapy. He applied moxa smoke to the skin above the larynx by waving burning moxa stick towards larynx for 15 to 20 minutes once a day, or alternatively had 3 to 8 inhalations of moxa smoke once a day (both methods have equal therapeutic effect and they are just two different ways of delivering moxa tar to the inflamed tissue in the larynx), continued for 15 days as a course. There was 3-day interval between two courses.

After two courses of treatment with moxibustion, the pain, dryness and swelling in the larynx started to ameliorate. With further 5-course treatment, all the pain, dryness and swelling in the larynx completely disappeared and the author was able to talk normally without any voice fatigue. The chronic inflammation in the larynx had been cured by moxibustion.

The author also used similar therapy to treat another 25 chronic laryngitis Patients.

Selection of patients: random.

Number of patients: 25

Gender of patients: female: 11, male: 14

The age of patient: 26 to 85 year old

Location of patients: 3 in Australia, 22 in China

Criteria of diagnosis: Diagnosis was based on clinical symptoms and signs (laryngoscopy is not reliable for diagnosis of chronic laryngitis, laryngoscopy examination could be normal but patients still had voice fatigue and symptoms in the larynx).

All patients had hoarse voice, pain, dryness and heaviness in the larynx and voice fatigue for more than 6 months without improvement.

Previous treatment: all patients had oral Prednisolone treatment from $25 \mathrm{mg}$ daily to $50 \mathrm{mg}$ daily for 7 to 30 days. None of them had response to Prednisolone treatment.

\section{Current treatment}

Method 1: Applying moxa smoke to the skin above the larynx for 15 to 20 minutes, one session a day or alternatively having 3 to 8 inhalations of moxa smoke, one session a day (both methods have equal therapeutic effect, one delivers moxa tar to larynx through skin absorption, another directly deposits moxa tar to the surface of vocal cord), 15 days is a course. There is a 3-day interval between two courses.

\section{2 patients received this therapy.}

Method 2: applying moxa-smoke-dissolved-water to the skin above the larynx 2 to 3 times a day, 15 days is a course, and there is 3 -day interval between two courses.

3 patients received this therapy.

Duration of treatment:

13 patients had 2 courses of treatment of method 1.

9 patients had 4 courses of treatment of method 1 .

3 patients had 4 courses of treatment of method 2 .

None of the 25 patients were allowed to take spicy food, smoking or drinking alcohol during their treatment.

Evaluation of therapeutic effect:

Evaluation of therapeutic effect is based on the clinical symptoms, signs and vocal function.

13 patients showed improvement in the pain, dryness and heaviness in the larynx and hoarse voice after 1 course of treatment of method 1. When they completed the 2nd course of treatment of method 1, all the pain, dryness and heaviness in the larynx and hoarse voice disappeared and could talk normally without any voice fatigue.

9 patients had improvement in the pain, dryness and heaviness and hoarse voice after 2 courses of treatment of method 1 . When they completed 4 courses of treatment of method 1, there was no pain, dryness or heaviness in the larynx, had no hoarse voice and could continue to talk for hours without any voice fatigue.

3 patients had improvement in the pain, dryness and heaviness in the larynx and hoarse voice after 2 courses of treatment of method 2 . 
When they completed 4 courses of treatment of method 2, they did not have any pain, dryness or heaviness in the larynx or hoarse voice and could talk as much as they wanted without any voice fatigue.

\section{Followed up}

Followed up for 3 months, no relapse.

The criteria of curing of chronic laryngitis: complete disappearance of symptoms (pain, dryness and heaviness in the larynx and sign (hoarse voice) of chronic laryngitis and returning to normal vocal function, i.e. talking without voice fatigue. The latter is the most accurate and important indicator of the efficacy of assessment for treatment of chronic laryngitis. If a tiny inflamed tissue left in the larynx, the chronic inflammation will rapidly flare up when the patient starts to talk.

No relapse for at least for 3 months unless the patient encounter the triggers again, e.g. virus infection, start heavy smoking or alcohol consumption.

According to the above criteria, the author considers all 25 chronic laryngitis patients have been cured by moxa tar therapy.

\section{Curing rate: $100 \%$}

Failure rate: 0

\section{$\mathrm{P}<0.001$.}

The above facts prove that the anti-inflammatory effect of the moxa tar can be reliably reproduced on different patients. The underlying mechanisms that sustain the chronic inflammation can be conquered by moxa tar.

The author draws a conclusion from this clinical experiment that moxa tar is a cure of chronic non-specific inflammation. This is of great significance because chronic laryngitis represents the incurability of chronic self-perpetuating inflammation. Chronic self-perpetuating inflammation is an important basic issue in pathology. Its importance is that it is involved in the pathogenesis of a wide range of chronic inflammatory diseases and it cannot be cured by any known antiinflammatory drugs. Curing chronic laryngitis means that chronic selfperpetuating inflammation can be cured. Its significance is far greater than curing of one particular chronic inflammatory disease. As many chronic inflammatory diseases share the same underlying mechanism as that of chronic laryngitis, regardless of the initiating stimuli or in what organ the inflammation occurs, the author infers that moxa tar can be used to treat other self-perpetuating chronic inflammatory diseases.

In chronic inflammation, the prolonged inflammation causes tissue damage. The inflammation becomes self-perpetuating i.e., the inflammation will last forever even when the initiating stimuli are eliminated (like in COPD and chronic gastritis). Lymphocyte (mainly $\mathrm{T}$ cell) infiltrate is a character of chronic inflammation and $\mathrm{T}$ cell plays an important role in sustaining chronic inflammation. There are complicated intercellar reactions between $\mathrm{T}$ cells and other cells (like macrophages in chronic laryngitis and COPD ..., neutrophils in Buerger's disease and COPD..., endothelial cells in atherosclerosis and eosinophils in asthma) in chronic inflammation. In these intercellar reactions, activated macrophages, neutrophils, eosinophils and endothelial cells produce interleukin 12 and other cytokines to activate T cells, in turn, activated T cells secrete interferon-gamma, interleukin 8 and other cytokines to activate macrophages, neutrophils, eosinophils, and endothelial cells, the results are positive feedback loops between these cells that fuel and sustain chronic inflammation.
Once these cycles of intercellar reactions are established, no medication can terminate these intercellar reactions. The real underlying mechanisms that sustain the self-perpetuating chronic inflammation could be much more complicated than we understand today. However, the therapeutic value of moxa tar is that it can disrupt the mechanisms that sustain chronic inflammation and cure selfperpetuating chronic inflammation.

Many chronic inflammatory diseases could have the same or similar underlying mechanisms and COPD (chronic obstructive pulmonary disease) is one of them, therefore the author generalized moxa tar treatment to COPD.

\section{Treatment of COPD}

COPD is a common and important chronic inflammatory disease. It is responsible for about $4.6 \%$ of total mortality worldwide and there are no effective preventative treatments currently available.

Current theory of pathogenesis of COPD is that cigarette smoking, air pollution and noxious agents can activate alveolar macrophage $\rightarrow$ the activated macrophage secretes various cytokines like interleukin 8 and interleukin 12 , these cytokines consequently activate neutrophil and CD $8+\mathrm{T}$ cell, inducing an inflammatory reaction. Activated neutrophils and macrophages secrete proteolytic enzymes like neutrophil elastase, macrophage elastase and matrix Metalloproteinases. These proteases cause mucus hypersecretion in small airway $\rightarrow$ chronic obstructive bronchitis and disruption of the wall of alveoli $\rightarrow$ emphysema; both conditions result in progressive deterioration of the lung function. The underlying inflammation in COPD causes relentless destruction of lung tissue and fibrosis of small airway. Neither steroid nor other new anti-inflammatory drugs (like phosphodiesterase- 4 inhibitors, nuclear factor-kappa B inhibitors) can stop the progression of COPD. As the chronic inflammation in COPD is also self-perpetuating like the inflammation in chronic laryngitis and moxa tar can cure the inflammation in chronic laryngitis, the author infers that moxa tar is able to treat and even cure the inflammation in COPD. The author treated 25 COPD patients with moxa tar and had good results.

Treatment of COPD with moxa tar (moxa smoke)

Number of patients: 25

Gender of patient: female: 5 male: 20

Age of patients: 50 - 88 year old

Location of patients: 24 patients were in China, 1 patient was in Australia.

Selection of patients: no active respiratory tract infection, cessation of smoking.

22 patients had a history of cigarette smoking for more than 10 years.

3 patients had no history of smoking

All cigarette smokers had stopped smoking for at least one year before receiving the inhalation therapy of moxa smoke.

Current medications: SABA (Short-Acting Beta2-Agonists), LABA (Long-Acting Beta2-Agonists), LAMA (Long-Acting Muscarinic Antagonists) and or ICS (Inhaled corticosteroids).

\section{Classification of patients}

All 25 patients: FEV1/FVC $<70 \%$ 
3 patients: FEV $1<30 \%$ predicted, had severe dyspnoea and cyanotic lips at rest, had no productive cough, classified as very severe emphysema patients.

10 patients: $30 \%$ predicted $<$ FEV $1<40 \%$ predicted, had dyspnoea on minimal exertion with or without chronic productive cough, classified as severe patients.

12 patients: $\mathrm{FEV} 1=40-59 \%$ predicted, had dyspnoea on moderate exertion with or without cough and/or sputum, classified as moderate severe patients.

\section{2 patients: diagnosed as emphysema by CT scan}

3 patients: had dyspnoea and productive cough for more than three consecutive months in more than two consecutive years without imaging evidence of emphysema, diagnosed as chronic obstructive bronchitis.

These 3 patients: $40 \%$ predicted $<$ FEV $1<59 \%$ predicted, classified as moderate severe chronic obstructive bronchitis patients.

All 25 patients still had mild to severe COPD symptoms (shortness of breath on exertion) with treatment of inhaled bronchodilators and / or ICS before receiving inhalation therapy of moxa smoke.

All 25 patients had moderate to severe COPD symptoms (from breathless walking on level ground to severe dyspnoea at rest) after one or two days of discontinuation of inhaled bronchodilators and/or ICS.

Pre-conditions for receiving treatment:

1. No evidence of active respiratory tract infection (no fever, no yellow or green colour sputum)

2. If patients did have active respiratory tract infection, they must be treated with antibiotics to clear the infection before starting the inhalation treatment.

\section{Stop smoking}

Treatment:

Method of treatment:

3-8 inhalations of moxa smoke per session a day

15 days as a course

Total treatments: 2-4 courses (there is a 3-5 day break between courses)

Alternative treatment (for those who cannot cope with smoke inhalation)

It is to apply the moxa smoke to the front and back of the chest except the precordial area by waving a burning moxa stick for $7-10$ minutes, one session a day.

15 days as a course

Total treatments: $2-4$ courses (there is a 3-5 day break between courses)

23 patients: treated with inhalation therapy of moxa smoke

2 patients: treated with alternative therapy (they could not cope with smoke inhalation)

Normally when patients start a new therapy, they should discontinue with the previous therapy, but COPD patients would have severe shortness of breath if they suddenly stop inhaled bronchodilators (SABA, LABA and LAMA) and or ICS, therefore these patients were allowed to keep their previous treatment with inhaled bronchodilators and or ICS when they started the inhalation therapy of moxa smoke or the alternative therapy if they had severe shortness of breath without inhalation of bronchodilators and or ICS. They continued with this cotreatment until the inhalation therapy or alternative therapy started to take effect i.e. they had less shortness of breath, compared with when only using bronchodilators and or ICS.

Then they stopped bronchodilators/ICS completely, but continued with inhalation therapy of moxa smoke until their COPD symptoms completely disappeared. The "wash out" period of the effect of SABA, LABA, LAMA and ICS is one day.

All 3 very severe patients and 10 severe patients had co-treatment initially.

7 of 12 moderate patients also had co-treatment until the inhalation therapy started to take effect.

5 of 12 moderate patients stopped previous treatment of inhaled bronchodilators and or ICS one day before they started inhalation therapy of moxa smoke. They managed to cope with shortness of breath after discontinuation of inhaled bronchodilators and or ICS by reducing physical activity.

Evaluation of the Therapeutic Effects of moxa tar treatment:

Clinical therapeutic effects were evaluated according to COPD symptoms and values of spirometry.

$3 / 25$ patients ( 1 chronic obstructive bronchitis patient, 2 moderate emphysema patients) had no improvement in their COPD symptoms ( shortness of breath) and spirometry values after having one course (15 days) treatment with the inhalation of moxa smoke and refused to continue treatment with this therapy.

$22 / 25$ patients showed improvement in breathlessness and or cough with or without sputum after 1 or 2 courses of treatment with inhalation of moxa smoke or alternative therapy.

20 of 22 improved Patients had co-treatment initially, at this stage they stopped treatment with bronchodilators or ICS but continued treatment with the inhalation therapy of moxa smoke or the alternative therapy for another 1 or 2 courses until symptoms free.

2 of 22 improved patients continued with mono-therapy (only inhalation of moxa smoke) for another course until symptoms free.

The lips cyanosis of 3 very severe emphysema patients has disappeared after 4 courses of treatment with inhalation therapy.

The criteria of symptoms free:

1. No breathlessness on moderate exertion for moderate severe and severe patients

2. No breathlessness on walking level ground for very severe patients

\section{No productive cough}

4. Do normal daily life activities without limitation

Comparison of spirometry values of 22 symptoms free patients before and after moxa tar treatment:

3 very severe and 10 severe emphysema patients: No change in 
spirometry value before and after moxa tar treatment.

7 moderate severe emphysema patients:

Before moxa tar treatment: FEV1/FVC $<70 \%$; FEV1 $=40-59 \%$ predicted.

After moxa tar treatment: FEV1/FVC $<70 \%$; FEV1 $=50-70 \%$ predicted.

2 moderate severe chronic obstructive bronchitis patients:

Before moxa tar treatment: FEV1/FVC $<70 \%$; FEV1 $=40-59 \%$ predicted.

After moxa tar treatment: FEV1/FVC <70\%; FEV1 > 80\% predicted

All these 22 patients became symptoms free after 2 to 4 courses of treatment with moxa tar. They could do their normal daily life activities without limitation and did not need any further treatment with SABA, LABA, LAMA and or ICS.

These patients were followed up from 6 months to 2 years, no relapse. All 22 patients kept symptoms free.

Spirometry tests were repeated for these 22 symptoms free patients from 6 months to 2 years after moxa tar treatment, the spirometry values of all 22 patients were the same as that when they had just finished moxa tar treatment.

At clinical level, moxa tar therapy show some curative effect for COPD patients, i.e., after a period of treatment with moxa tar, 22 of 25 COPD patients kept symptoms free without any further treatment.

Symptoms free rate is $88 \%$.

$\mathrm{P}<0.01$

3 patients had no improvement in their COPD symptoms or spirometry values after one course ( 15 days) treatment with inhalation of moxa smoke.

Failure rate: $12 \%$

Self-comparison of these 25 patients showed big difference in their COPD symptoms before and after moxa tar treatment.

Before moxa tar treatment, all 25 patients needed daily treatment with inhaled bronchodilators and or ICS, but they still had mild to severe shortness of breath and or cough with or without sputum; without treatment with inhaled bronchodilators or ICS, all these 25 patients had moderate to severe dyspnoea and or cough with or without sputum, their daily life activities were seriously affected by COPD.

After moxa tar treatment, 22 of 25 patients kept symptoms free without any further treatment.

The difference of COPD symptoms of these 22 of 25 patients before and after moxa tar treatment is significant.

As the inflammation in COPD is intimately linked with COPD symptoms, the author presumes that the disappearance of COPD symptoms from these 22 of 25 patients could be due to curing of the inflammation in the lungs and respiratory tract. This would correct the ventilation perfusion mismatch in the lungs of these patients and significantly increase the efficiency of gas-exchanging in the lungs, resulting in COPD symptoms free in these patients though there is no change or only slightly change in the results of lung function tests. But this presumption needs to be validated by more objective tests, like diffusing capacity for carbon monoxide, bronchoalveolar lavage for counting inflammatory cells, or bronchoscopic biopsy for pathology before and after treatment.

The author assumes that the reason that there is no improvement in the Spirometry values of severe and very severe patients after treatment with moxa tar is that the tissue damage in the lungs of these patients cannot be reversed though the inflammation could be cured by moxa tar. Curing of inflammation in the lungs can stop the progression of COPD but cannot reverse the lung tissue damage.

\section{Discussion}

The results of treatment of COPD with moxa tar are very encouraging. At clinical level, moxa tar shows some curative effect for COPD, which has never been shown by any other available drug therapies. Comparing the inflammation in COPD to the inflammation in chronic laryngitis, there are some similarities. In both diseases there is ongoing inflammation when the initiating stimuli are eliminated, i.e. the inflammation is self-perpetuating. The author needs to point out that from the point of view of pathology, it is much more difficult to treat the inflammation in chronic laryngitis than the inflammation in COPD. The inflammation in COPD is partially resistant to corticosteroid (treatable but not curable) and it can be suppressed by corticosteroid to some extent though it cannot be cured by corticosteroid. But the inflammation in chronic laryngitis is completely resistant to corticosteroid and it is not treatable by any current antiinflammatory drugs. As moxa tar is definitely a cure for the chronic inflammation in chronic laryngitis and the inflammation in both conditions is self-perpetuating (the author believes that the underlying mechanisms that sustain the chronic inflammation in both conditions are the same), the author presumes that it could also be a cure for COPD. Moxibustion has also been used to treat eczema, allergic dermatitis, diabetic foot, angina pectoris, diabetic foot, inflammatory bowel diseases, thromboangiitis obliteran (Burger's disease), psoriasis, asthma, chronic muscle injury, pulmonary fibrosis ...... by Chinese Herbalists and it has been proved that it is a very effective therapy for these chronic inflammatory diseases. Since in all above-mentioned and many other chronic inflammatory diseases $\mathrm{T}$ cell plays a pivotal role in sustaining the chronic inflammation, the author hypothesizes that moxa tar has the potential to treat any $\mathrm{T}$ cell involved chronic inflammatory diseases and its anti-inflammatory property could be due to inhibiting $\mathrm{T}$ cell activity or $\mathrm{T}$ cell related molecules.

As moxa tar has potent and unique anti-inflammatory property, the author thinks it is an important and promising source for developing new anti-inflammatory drug. If a new anti-inflammatory drug can be made from moxa tar, it will lead to a breakthrough in treating many incurable chronic inflammatory diseases, like COPD, multiple sclerosis and atherosclerosis. The therapeutic potential of moxa tar should be thoroughly studied by molecular biologists.

\section{End Notes}

1. Meridian line: The term "meridian lines" is used in a number of natural therapies with acupuncture being perhaps the best known. Meridian lines are invisible lines that carry energy throughout the body. Along these lines are points called meridian points. Meridian lines cannot be seen or felt like other systems in the body such as the circulatory or nervous system. When a person is in good (balanced) health, their meridian lines will be open and clear of blockages.

\section{References}

1. Barnes PJ (2003) Chronic obstructive pulmonary disease * 12: New treatments for COPD. Thorax 58: 803-808. [Crossref] 
2. V Kumr, Al Abbas, N Fausto, R Mitchell, C. Alpers, et al. (2007) Robbins Basic Pathology Saunders, an imprint of Elsevier Inc, Philadelphia, PA.

3. Rubin R, Strayer D (2008) Rubin's Pathology, clinicopathologic Foundations of Medicine. (5th edtn). Wolters Kluwer, Philadelphia, USA.

4. Fauci A, Braunwald E, Kasper D, Hauser S, Longo D (2008) Harrison's Principles of Internal Medicine. (17th edtn). McGraw-Hill Companies, New York, United States.

5. Li R, Li WJ, Cai YN, Li ZG, Luo Q, et al. (2005) Effects of Moxibustion at FeiShu and Gaohuang on expression of TGF-beta1 in the Bleomycin-induced pulmonary fibrosis. Zhongguo Zhen Jiu 25: 790-792.

6. Dykewicz MS (2001) Newer and alternative non-steroidal treatments for asthmatic inflammation. Allergy Asthma Proc 22: 11-15. [Crossref]

7. Frew AJ, Plummeridge MJ (2001) Alternative agents in asthma. J Allergy Clin Immunol 108: 3-10. [Crossref]

8. Legg J, Warner J (2000) Asthma--the changing face of drug therapy. Indian $J$ Pediatr 67: 147-153. [Crossref]
9. Ribas DC, Monte CR, Garcia BM, Besoli MA, Soler MC (1999) Efficacy of methotrexate in the treatment of corticosteroid dependent asthmatic patients. Rev Clin Esp199: 142-146.

10. Kazimierczak A, Maziarka D, Skorupa W, KuÅ> J (1997) [Use of methotrexate for treatment of corticosteroid-dependent asthma]. Pneumonol Alergol Pol 65: 225-230. [Crossref]

11. Aono Y, Nishioka Y, Inayama M, Ugai M, Kishi J, et al. (2005) Imatinib as a nove antifibrotic agent in bleomycin-induced pulmonary fibrosis in mice. Am J Respir Crit Care Med 171: 1279-1285.

12. Arai T, Abe K, Matsuoka H, Yoshida M, Mori M, et al. (2000) Introduction of the interleukin-10 gene into mice inhibited bleomycin-induced lung injury in vivo. Am J Physiol Lung Cell Mol Physiol 278: L914- L922.

13. Kumar V, Abbas A, Fausto N, Mitchell R, Alpers C, et al. (2007) Robbins Basic Pathology, (8thedn), Saunders, Philadelphia, PA, USA, 343-353.

Copyright: $\mathbb{C} 2017$ Zeng Q. This is an open-access article distributed under the terms of the Creative Commons Attribution License, which permits unrestricted use, distribution, and reproduction in any medium, provided the original author and source are credited. 